\title{
Coronary artery assessment by multidetector computed tomography in patients with prosthetic heart valves
}

\author{
Jesse Habets • Renee B. A. van den Brink • \\ Ruben Uijlings • Anje M. Spijkerboer • \\ Willem P. Th. M. Mali • Steven A. J. Chamuleau • \\ Ricardo P. J. Budde
}

Received: 21 July 2011 /Revised: 10 November 2011 / Accepted: 16 November 2011 /Published online: 23 December 2011

(C) The Author(s) 2011. This article is published with open access at Springerlink.com

\begin{abstract}
Objectives Patients with prosthetic heart valves may require assessment for coronary artery disease. We assessed whether valve artefacts hamper coronary artery assessment by multidetector CT.

Methods ECG-gated or -triggered CT angiograms were selected from our PACS archive based on the presence of prosthetic heart valves. The best systolic and diastolic axial reconstructions were selected for coronary assessment. Each present coronary segment was scored for the presence of valve-related artefacts prohibiting coronary artery assessment. Scoring was performed in consensus by two observers.

Results Eighty-two CT angiograms were performed on a 64-slice $(n=27)$ or 256-slice $(n=55)$ multidetector CT. Eighty-nine valves and five annuloplasty rings were present. Forty-three out of 1160 (3.7\%) present coronary artery segments were non-diagnostic due to valve artefacts $(14 / 82$
\end{abstract}

J. Habets $(\bowtie) \cdot$ W. P. T. M. Mali • R. P. J. Budde

Department of Radiology, University Medical Center Utrecht,

Heidelberglaan 100, E01.132, PO BOX 85500, 3508 GA Utrecht,

The Netherlands

e-mail: J.Habets@umcutrecht.nl

R. B. A. van den Brink

Department of Cardiology, Academic Medical Center,

Amsterdam, The Netherlands

R. Uijlings

Department of Cardiology, Deventer Hospital,

Deventer, The Netherlands

\section{A. M. Spijkerboer}

Department of Radiology, Academic Medical Center,

Amsterdam, The Netherlands

S. A. J. Chamuleau

Department of Cardiology, University Medical Center,

Utrecht, The Netherlands patients). Valve artefacts were located in right coronary artery $(15 / 43 ; 35 \%)$, left anterior descending artery $(2 / 43$; $5 \%)$, circumflex artery $(14 / 43 ; 32 \%)$ and marginal obtuse $(12 / 43 ; 28 \%)$ segments. All cobalt-chrome containing valves caused artefacts prohibiting coronary assessment. Biological and titanium-containing valves did not cause artefacts except for three specific valve types.

Conclusions Most commonly implanted prosthetic heart valves do not hamper coronary assessment on multidetector CT. Cobalt-chrome containing prosthetic heart valves preclude complete coronary artery assessment because of severe valve artefacts.

Key Points

- Most commonly implanted prosthetic heart valves do not hamper coronary artery assessment

- Prosthetic heart valve composition determines the occurrence of prosthetic heart valve-related artefacts

- Björk-Shiley and Sorin tilting disc valves preclude diagnostic coronary artery segment assessment

Keywords Computed tomography Prosthetic heart valve . Coronary artery disease $\cdot$ Coronary artery $\cdot$ Artefacts

\section{Introduction}

The introduction of ECG-gated and triggered multidetector computed tomography (MDCT) technology has resulted in new diagnostic cardiac applications, most noticeably coronary artery assessment [1]. Recently, MDCT has also shown potential for the evaluation of prosthetic heart valve (PHV) (dys)function by providing complementary diagnostic information to echocardiography and fluoroscopy, especially in patients with suspected PHV obstruction and PHV endocarditis [2-6]. 
Besides evaluation of the exact cause of PHV dysfunction, MDCT may have complementary clinical value for the surgical planning in patients considered for reoperation after previous PHV implantation. MDCT can be used to evaluate the presence of coronary artery disease (CAD) and the patency of present bypass grafts to serve as an alternative for classical coronary angiography, and the distance between the sternum and the right ventricle can be measured [7]. Patients with a normal functioning PHV may also be candidates for $\mathrm{CT}$ assessment of the coronary arteries in newly developed angina.

Each PHV type has its own specific imaging characteristics on CT $[8,9]$. Most PHVs have limited artefacts but at present little is known about which PHV types and positions induce artefacts to such an extent that they prohibit coronary assessment on MDCT. Knowledge of which PHV types and positions prohibit coronary assessment is clinically important to determine whether adequate CT assessment of coronary segments is to be expected or that the patient should be referred for conventional coronary angiography straight away. The purpose of this study was to determine which PHV types induce artefacts that hamper coronary artery segment assessment on MDCT in a cohort of patients with a PHV in whom an ECG-gated or -triggered PHV had been performed.

\section{Materials and methods}

\section{CT angiogram selection}

We reviewed all ECG-gated or prospectively triggered PHV $\mathrm{CT}$ angiograms (CTAs) performed in the University Medical Center Utrecht and Academic Medical Center Amsterdam between 2003 and September 2011 for the presence of PHVs that were imaged in both systolic and diastolic phase. We excluded CTAs that included only systolic or diastolic imaging, were performed on 16-slice MDCT, non-contrast enhanced imaging as well as CTAs in patients enrolled in an ongoing prospective diagnostic cross-sectional study on CT PHV assessment. Furthermore, patients with multiple CTA examinations were included only once. Patients with a concomitant mitral or tricuspid annuloplasty ring were included.

CTAs were performed on 64 or 256 slice MDCT systems (Brilliance 64 and iCT, Philips Medical Systems, Cleveland, Ohio). Contrast agents [Ultravist (iopromide) - $300 \mathrm{mg} \mathrm{I} / \mathrm{mL}$, Bayer Schering Pharma AG, Berlin, Germany or Iomeron (iomeprol) - $400 \mathrm{mg} \mathrm{I} / \mathrm{mL}$, Bracco UK Limited, London, United Kingdom] were administered in all patients. Cardiac CTAs were performed with a triphasic or dual phasic contrast injection protocol. The triphasic protocol started with a $100 \%$ contrast injection (phase 1 ) followed by a $30 \% / 70 \%$ contrast/ saline mixture (phase 2), and concluded with a saline flush (phase 3). Contrast volumes were adjusted for the patients body weight with an iodine flow of $1.6 \mathrm{~g} / \mathrm{s}$ for patients $<70$ kilogram $(\mathrm{kg}), 1.8 \mathrm{~g} / \mathrm{s}$ for patients $70-85 \mathrm{~kg}$ and $2.0 \mathrm{~g} / \mathrm{s}$ for patients $>85 \mathrm{~kg}$. The dual phase contrast protocol consisted of a contrast injection of 100 millilitre $(\mathrm{mL})$ followed by a saline flush. For aortic CTAs, a fixed bolus of $100 \mathrm{ml}$ was followed by a saline flush of $50 \mathrm{ml}$. In general, injection flow was set to 5-6 ml/s. The CTA selection resulted in the inclusion of 82 patients (48 males, 34 females) with 94 PHVs and annuloplasty rings. The mean age of our study population was $58 \pm$ 14 years (mean $\pm \mathrm{SD}$ ). All 82 patients underwent CTA examinations on 64-slice $(n=27)$ or 256-slice $(n=55)$ MDCT systems. The indications for CTA examinations were: PHV dysfunction $(n=47)$, aortic aneurysm evaluation $(n=17)$, aortic dissection $(n=8)$, coronary assessment $(n=3)$, cardiac other $(n=5)$ and other $(n=2)$. In four of 82 patients $(5 \%)$ betablockers were administered to lower the heart rate. Nitroglycerin was not routinely administered in our patients.

CT data were retrieved from the PACS archive of the Radiology department and sent to a dedicated workstation for image analysis. Heart rate during imaging was obtained from the CT data. DLP values were obtained if dose information was available. DLP values were converted to radiation exposure (mSv) by using 0.017 for cardiac CTAs, and 0.016 (mean of 0.017 for the chest and 0.015 for the abdomen) for thoraco-abdominal aortic CTAs as conversion factor. Patient and PHV data were obtained from the patient's medical files. The study was performed under a waiver from the institutional reviewing board. Data on the image quality of the PHV itself in a number of these patients have been previously published [9].

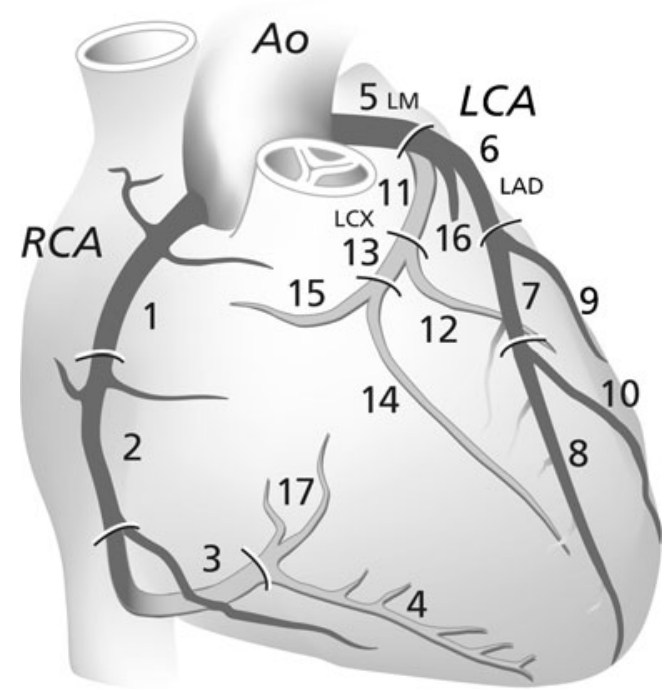

Fig. 1 Coronary artery segments according to the American Heart Association classification [10]. Ao $=$ Aorta; RCA $=$ Right Coronary Artery; LCA = Left Coronary Artery; LM = Left Main branch; LAD = Left Anterior Descending Coronary Artery; LCX $=$ Left Circumflex Coronary Artery 
Table 1 Presence of valve-related artefacts classified per prosthetic heart valve (PHV) manufacturer and position

\begin{tabular}{|c|c|c|c|c|c|c|c|}
\hline \multirow[t]{2}{*}{ Manufacturer } & \multirow[t]{2}{*}{ Type } & \multirow[t]{2}{*}{ PHV type } & \multicolumn{5}{|c|}{ Number per valve position (number of valves that cause PHV-related artefacts) } \\
\hline & & & Aortic & Mitral & Pulmonary & Tricuspid & Total \\
\hline Carbomedics & Mechanical & Bileaflet & $21(0)$ & $3(1)$ & $0(0)$ & $0(0)$ & $24(1)$ \\
\hline St Jude & Mechanical & & $8(2)$ & $4(3)$ & $0(0)$ & $0(0)$ & $12(5)$ \\
\hline $\mathrm{ON}-\mathrm{X}$ & Mechanical & & $5(0)$ & $0(0)$ & $0(0)$ & $1(0)$ & $6(0)$ \\
\hline Sorin bileaflet & Mechanical & & $5(3)$ & $1(0)$ & $1(1)$ & $0(0)$ & $7(4)$ \\
\hline Duromedics & Mechanical & & $0(0)$ & $1(1)$ & $0(0)$ & $0(0)$ & $1(1)$ \\
\hline Medtronic Hall & Mechanical & Tilting disc & $9(0)$ & $3(0)$ & $0(0)$ & $0(0)$ & $12(0)$ \\
\hline Sorin monoleaflet & Mechanical & & $1(1)$ & $1(1)$ & $0(0)$ & $0(0)$ & $2(2)$ \\
\hline Björk-Shiley & Mechanical & & $3(3)$ & $1(1)$ & $0(0)$ & $0(0)$ & $4(4)$ \\
\hline $\mathrm{CE}$ & Annuloplasty ring & & $0(0)$ & $4(0)$ & $0(0)$ & $1(0)$ & $5(0)$ \\
\hline Perimount & Biological & & $15(0)$ & $0(0)$ & $0(0)$ & $0(0)$ & $15(0)$ \\
\hline Mitroflow & Biological & & $1(0)$ & $0(0)$ & $0(0)$ & $0(0)$ & $1(0)$ \\
\hline Medtronic mosaic & Biological & & $2(0)$ & $0(0)$ & $0(0)$ & $0(0)$ & $2(0)$ \\
\hline Freestyle & Biological & & $1(0)$ & $0(0)$ & $0(0)$ & $0(0)$ & $1(0)$ \\
\hline Epic St Jude & Biological & & $2(0)$ & $0(0)$ & $0(0)$ & $0(0)$ & $2(0)$ \\
\hline Total & & & $73(9)$ & $18(7)$ & $1(1)$ & $2(0)$ & $94(17)$ \\
\hline
\end{tabular}

The number of PHVs causing artefacts prohibiting assessment of at least one coronary artery segment are presented in parentheses

Table 2 Coronary artery segments and valve-related artefacts specified per prosthetic heart valve (PHV) manufacturer type in aortic position

\begin{tabular}{|c|c|c|c|c|}
\hline PHV position & PHV manufacturer & PHV type & $\begin{array}{l}\text { Present segments*/ } \\
\text { non-assessable due } \\
\text { to PHV artefacts }\end{array}$ & $\begin{array}{l}\text { Non-diagnostic coronary } \\
\text { segments due to PHV } \\
\text { artefacts }\end{array}$ \\
\hline & Carbomedics & & $307 / 0$ & None \\
\hline \multirow[t]{18}{*}{ Aortic } & St Jude & Bileaflet & $117 / 2$ & $2 \times$ RCA segment 1 \\
\hline & Sorin & & $77 / 3$ & $2 \times$ RCA segment 1 \\
\hline & & & & $1 \times$ LCX segment 11 \\
\hline & $\mathrm{ON}-\mathrm{X}$ & & $69 / 0$ & None \\
\hline & Medtronic Hall & Tilting disc & $127 / 0$ & None \\
\hline & Björk-Shiley & & $34 / 11$ & $3 \times \mathrm{RCA}$ segment 1 \\
\hline & & & & $3 \times$ RCA segment 2 \\
\hline & & & & $1 \times$ LCX segment 11 \\
\hline & & & & $1 \times \mathrm{MO}$ segment 12 \\
\hline & & & & $2 \times$ LCX segment 13 \\
\hline & & & & $1 \times \mathrm{MO}$ segment 14 \\
\hline & Sorin & & $16 / 2$ & $1 \times$ RCA segment 1 \\
\hline & & & & $1 \times$ RCA segment 2 \\
\hline & CE Perimount & Biological & $218 / 0$ & None \\
\hline & Medtronic Mosaic & & $31 / 0$ & None \\
\hline & Freestyle & & $15 / 0$ & None \\
\hline & Mitroflow & & $16 / 0$ & None \\
\hline & Epic St Jude & & $27 / 0$ & None \\
\hline
\end{tabular}

\footnotetext{
* Total number of present segments in Tables 1,2 and 3 is higher than 1160 owing to multiple assessment of coronary segments in patients with multiple PHVs as the segments are presented for each PHV separately
} 
Image analysis

Assessment of the MDCT was performed on a dedicated workstation (Extended Brilliance Workstation, Philips Healthcare, Best, The Netherlands). The best systolic and diastolic image phase in the retrospectively gated or prospectively triggered $\mathrm{CT}$ dataset for coronary assessment was selected. Coronary arteries were assessed according to the 17-segment modified American Heart Association classification [10] on the axial images (Fig. 1).

First, each coronary segment was scored as present or absent. Absent was defined as no visible segment, but sufficient high image quality to exclude non-visibility of other causes or a segment not included in the imaging range. For present segments, the image quality was scored on a three-point scale ( $1=$ non-diagnostic, $2=$ acceptable, $3=$ good). The criteria for the different scores were formulated as follows: (1) non-diagnostic: segment details not sufficient visualised to perform diagnostic assessment; (2) acceptable: adequate segment details with limited artefacts; and (3) good: perfect segment details without artefacts. Reasons for non-diagnostic segments were classified as: valve- or ring annuloplasty-related artefacts, or other (e.g. motion, small vessel size, low contrast enhancement and pacemaker lead artefacts). Scoring was performed in consensus by two observers ( $\mathrm{JH}$ and $\mathrm{RB}$ ) with 2 years and 5 years experience in CT coronary assessment, respectively.

Data analysis

Data analysis was restricted to descriptive statistics. Data were presented as means \pm standard deviation (SD) for continuous data with a parametric data distribution. Categorical data were presented in total numbers and/or percentages. The presence of PHV-related artefacts precluding coronary artery segment assessment was determined for

Table 3 Coronary artery segments and valve-related artefacts specified per PHV manufacturer type in other positions

\begin{tabular}{|c|c|c|c|c|}
\hline PHV position & PHV manufacturer & PHV type & $\begin{array}{l}\text { Present segments*/ } \\
\text { non-assessable due } \\
\text { to PHV artefacts }\end{array}$ & $\begin{array}{l}\text { Non-diagnostic coronary } \\
\text { segments due to PHV } \\
\text { artefacts }\end{array}$ \\
\hline \multirow[t]{23}{*}{ Mitral } & \multirow{5}{*}{ St Jude } & \multirow[t]{9}{*}{ Bileaflet } & $46 / 1$ & $1 \times$ LCX segment 13 \\
\hline & & & $51 / 6$ & $1 \times$ LCX segment 11 \\
\hline & & & & $1 \times$ MO segment 12 \\
\hline & & & & $1 \times$ LCX segment 13 \\
\hline & & & & $3 \times$ MO segment 14 \\
\hline & \multirow[t]{4}{*}{ Duromedics } & & \multirow[t]{4}{*}{$11 / 4$} & $1 \times$ LCX segment 11 \\
\hline & & & & $1 \times$ MO segment 12 \\
\hline & & & & $1 \times$ LCX segment 13 \\
\hline & & & & $1 \times$ MO segment 14 \\
\hline & Sorin & & $15 / 0$ & None \\
\hline & CE Mitral ring & Annuplasty ring & $62 / 0$ & None \\
\hline & Medtronic Hall & Tilting disc & $46 / 0$ & None \\
\hline & \multirow[t]{6}{*}{ Björk-Shiley } & & \multirow[t]{6}{*}{$14 / 6$} & $1 \times \mathrm{RCA}$ segment 2 \\
\hline & & & & $1 \times$ RCA segment 3 \\
\hline & & & & $1 \times$ LAD segment 8 \\
\hline & & & & $1 \times \mathrm{MO}$ segment 12 \\
\hline & & & & $1 \times$ LCX segment 13 \\
\hline & & & & $1 \times \mathrm{MO}$ segment 14 \\
\hline & \multirow[t]{5}{*}{ Sorin } & & \multirow[t]{5}{*}{$16 / 5$} & $1 \times$ LAD segment 8 \\
\hline & & & & $1 \times$ LCX segment 11 \\
\hline & & & & $1 \times$ MO segment 12 \\
\hline & & & & $1 \times$ LCX segment 13 \\
\hline & & & & $1 \times \mathrm{MO}$ segment 14 \\
\hline Pulmonary & Sorin & Bileaflet & $15 / 1$ & $1 \times$ RCA segment 2 \\
\hline \multirow[t]{2}{*}{ Tricuspid } & $\mathrm{ON}-\mathrm{X}$ & Bileaflet & $15 / 0$ & None \\
\hline & CE Tricuspid ring & Annuplasty ring & $15 / 0$ & None \\
\hline
\end{tabular}

${ }^{*}$ Total number of present segments in Tables 1, 2 and 3 is higher than 1160 owing to multiple assessment of coronary segments in patients with multiple PHVs as the segments are presented for each PHV separately 
Fig. 2 Distribution of nondiagnostic coronary segments in patients with prosthetic heart valves

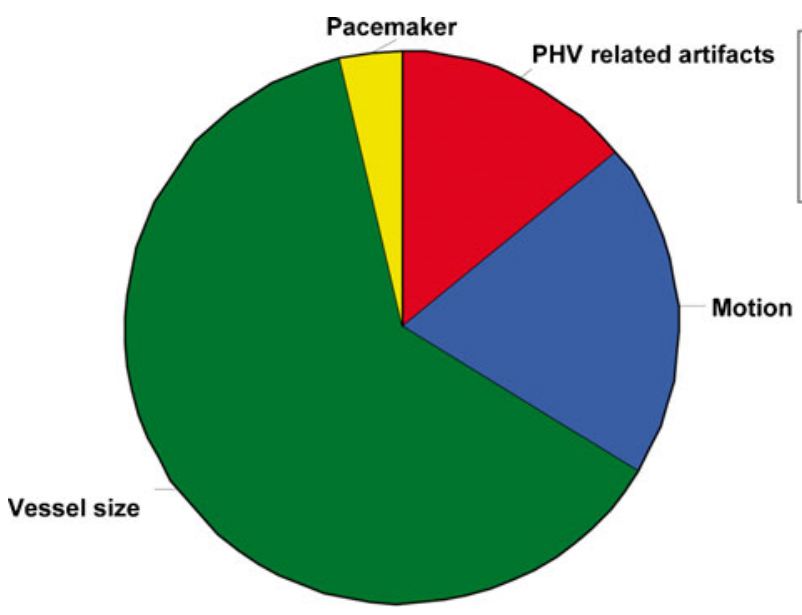

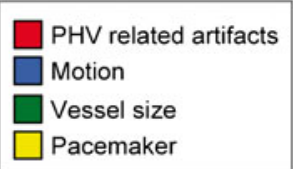

$\square$ PHV related artifacts
$\square$ Motion
$\square$ Vessel size
$\square$ Pacemaker

otion the different PHV types and PHV positions. These PHVrelated artefacts are presented per coronary artery segment.

\section{Results}

Mean heart rate during the 82 CTA examinations was $73 \pm 18$ beats per minute (bpm). In 15 of the 82 patients (18\%), the heart rate during $\mathrm{CT}$ data acquisition was missing. Mean heart rates were $73 \pm 18 \mathrm{bpm}$ and $72 \pm 19 \mathrm{bpm}$ for the mechanical PHV group and biological PHV group, respectively. Sixty of $82(73 \%)$ CT acquisitions were performed as a dedicated cardiac CTA including 51 retrospectively ECG-gated CTAs $(120 \mathrm{kV}, \geq 600 \mathrm{mAs})$ and nine prospectively triggered CTAs (120 kV, 200-250 mAs); and 22/82 (27\%) CTAs as a retrospectively ECG-gated CTA of the thoraco-(abdominal) aorta with a lower tube current (200-400 mAs). Radiation dose information was available for $42 / 82$ patients (51\%). Mean radiation dose for retrospectively ECG-gated cardiac CTAs was $15.6 \pm 5.5 \mathrm{mSv}$, and for thoraco-abdominal aortic CTAs $17.6 \pm 4.2 \mathrm{mSv}$. Ninety-four PHVs and annuloplasty rings of
14 different PHV and annuloplasty ring types were present: 68 mechanical PHVs (72\%), 21 biological PHVs (22\%), and 5 annuloplasty rings (5\%) (mitral $n=4$ and tricuspid $n=1$ ). PHVs and annuloplasty rings were positioned in the aortic $(n=73 ; 78 \%)$, mitral $(n=18 ; 19 \%)$, pulmonary $(n=1 ; 1 \%)$ and tricuspid $(n=2 ; 2 \%)$ positions (Table 1$)$.

PHV-related artefacts interfering with coronary artery assessment on MDCT

In total 17/94 (18\%) PHVs and annuloplasty rings induced artefacts prohibiting assessment of at least one coronary segment (Table 1). Valve-related artefacts per coronary segment are shown in Tables 2 and 3.

A theoretical total of 1394 coronary segments (82 patients $\times 17$ segments) were available for analysis. Two hundred and thirty-four of the 1394 segments $(17 \%)$ were scored absent. In total 1160 coronary segments were present for assessment. Three-hundred and six of 1160 coronary segments $(26 \%)$ demonstrated a non-diagnostic image quality, 538/1160 (46\%) had acceptable image quality; and in
Fig. 3 a Sorin tilting disc Prosthetic Heart Valve (PHV) in the aortic position, which causes PHV-related artefacts in RCA segment 1. b ON-X bileaflet Prosthetic Heart Valve (PHV) in the aortic position, which causes no PHV-related artefacts in RCA segment 1
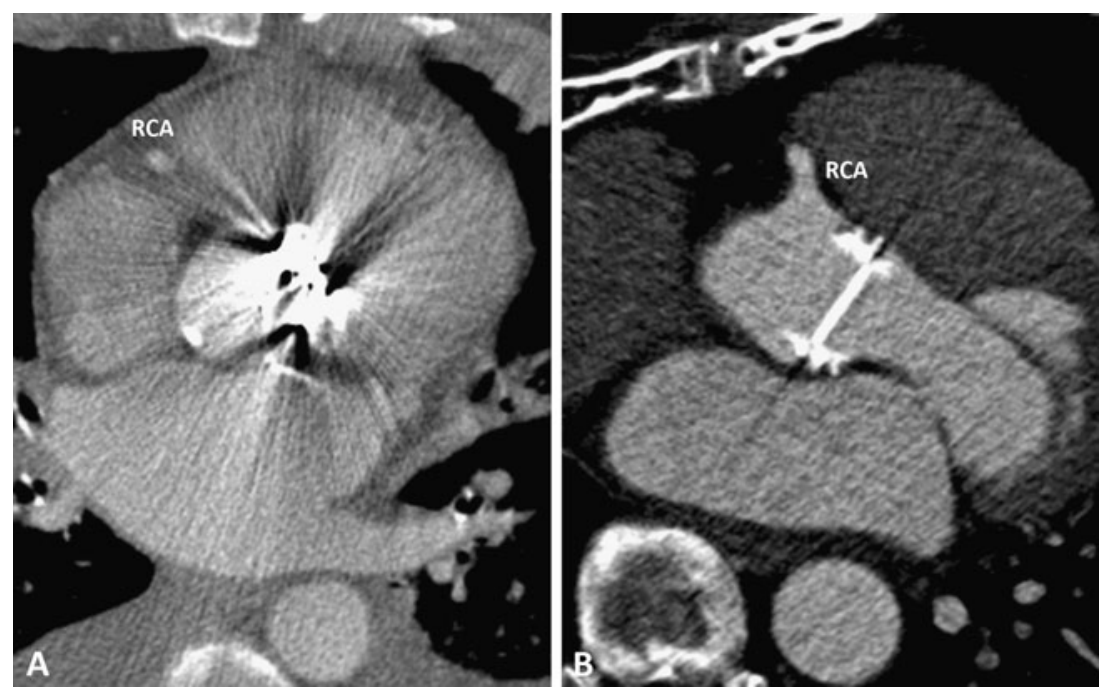


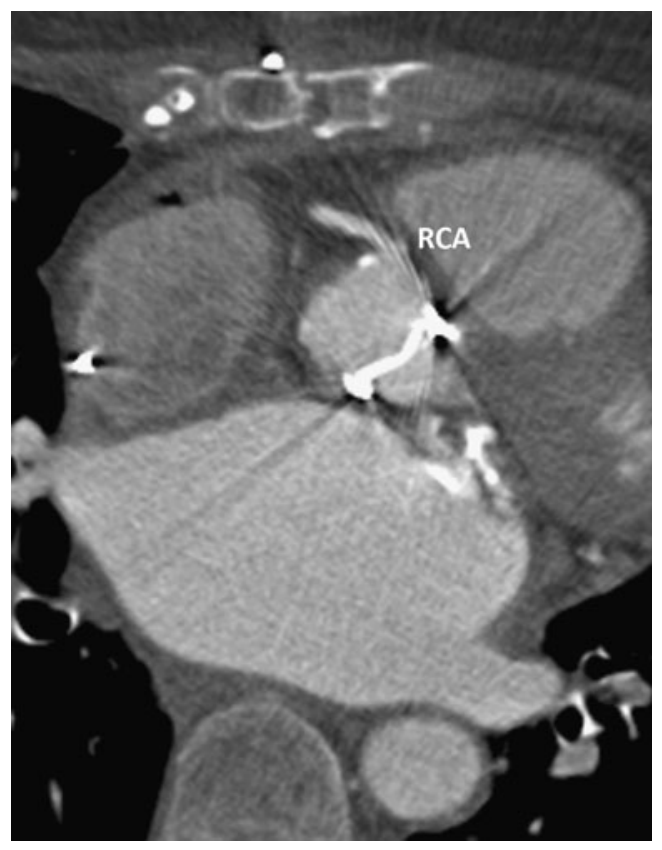

Fig. 4 St Jude bileaflet Prosthetic Heart Valve (PHV) in the aortic position, which causes PHV-related artefacts in RCA segment 1

$316 / 1160$ (27\%) image quality was good. The reasons for non-diagnostic image quality were PHV-related artefacts ( $n=43 ; 14 \%)$, motion ( $n=60$ segments; $20 \%)$, vessel size $(n=192 ; 63 \%)$; and pacemaker artefacts $(n=11 ; 4 \%)$ (Fig. 2). In patients with more than one PHV implanted, no coronary segments were non-assessable owing to artefacts originating from both valves.

None of the biological PHVs $(0 / 21 ; 0 \%)$ and annuloplasty rings $(0 / 5 ; 0 \%)$, and none of the ON-X $(0 / 6 ; 0 \%)$ and Medtronic Hall $(0 / 12 ; 0 \%)$ mechanical PHVs caused artefacts prohibiting coronary assessment.
In the aortic position, St Jude $(2 / 8 ; 25 \%)$ and Sorin $(3 / 5$; $60 \%$ ) bileaflet PHVs caused artefacts prohibiting coronary assessment, mainly of the proximal right coronary artery (RCA). The Björk-Shiley $(n=3 ; 100 \%)$ and Sorin tilting disc PHVs $(n=1 ; 100 \%)$ caused severe artefacts in RCA segments. Moreover, the Björk-Shiley tilting disc also caused artefacts in the left circumflex (LCX) and obtuse marginal (MO) branches (Table 2). The commonly implanted PHVs (Carbomedics bileaflet $(n=21 ; 0 \%)$ and Medtronic Hall tilting disc $(n=9 ; 0 \%)$ demonstrated no PHV-related artefacts in the aortic position (Tables 1 and 2). Figures 3 and 4 illustrate aortic PHVs with and without PHV-related artefacts in the RCA.

In the mitral position, Carbomedics $(1 / 3 ; 33 \%)$, St Jude $(3 / 4 ; 75 \%)$ and the Duromedics $(1 / 1 ; 100 \%)$ bileaflet PHVs caused artefacts prohibiting assessment of at least one coronary segment in the LCX and MO branches. Both BjörkShiley and the Sorin tilting disc PHVs caused severe artefacts in LCX and MO segments. The Björk-Shiley tilting disc also caused severe artefacts in RCA segments. Affected segments are detailed in Table 3. Figure 5 illustrates mitral PHVs with and without artefacts in the LCX. In the pulmonary position, the Sorin bileaflet PHV $(n=1 ; 100 \%)$ demonstrated PHVrelated artefacts in RCA segment 2. In the tricuspid position, ON-X bileaflet PHV $(n=1)$ and tricuspid annuplasty ring $(n=1)$ showed no PHV-related artefacts (Table 3).

\section{PHV related artefacts in different imaging phases}

In all patients, coronary artery segments were assessed in the best systolic and diastolic imaging phase. In the aortic position, four patients $(5 \%)$ demonstrated PHV-related artefacts precluding diagnostic coronary assessment of RCA segment 1 only in diastolic $(n=2 ; 50 \%)$ or systolic $(n=2 ; 50 \%)$ phase. In the mitral position, one patient $(6 \%)$ had only PHV-related artefacts in the diastolic phase in the LCX and MO branches.
Fig. 5 a Björk-Shiley tilting disc Prosthetic Heart Valve (PHV) in the mitral position, which causes PHV-related artefacts in LCX. b Medtronic Hall tilting disc PHV in the mitral position, which causes no PHV-related artefacts in LCX
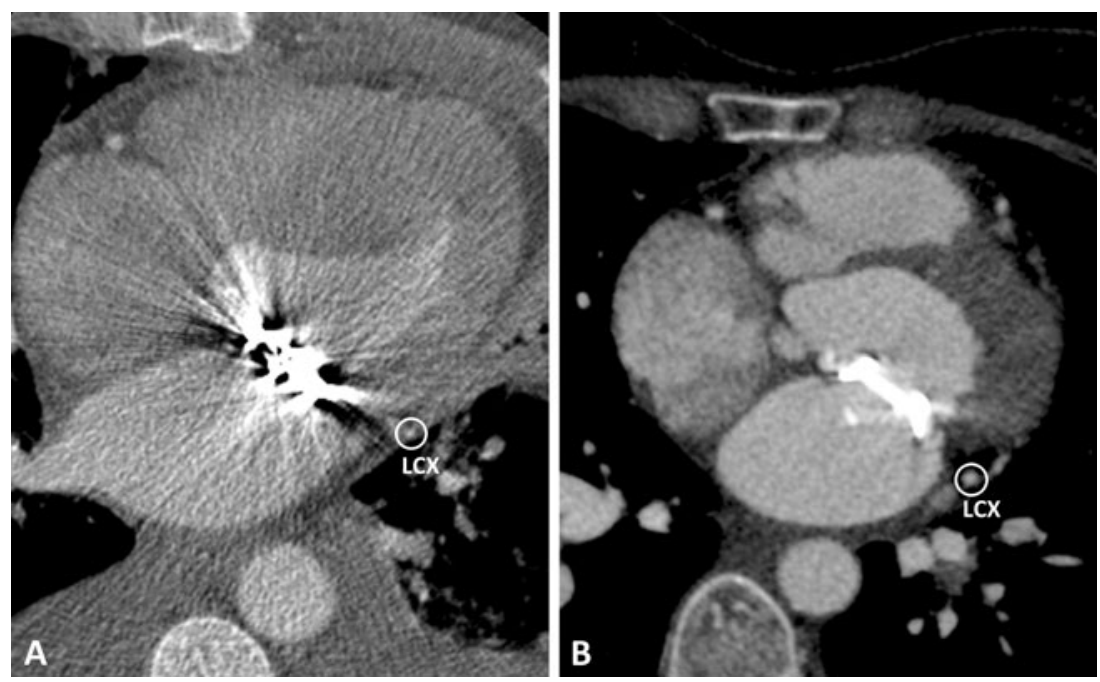


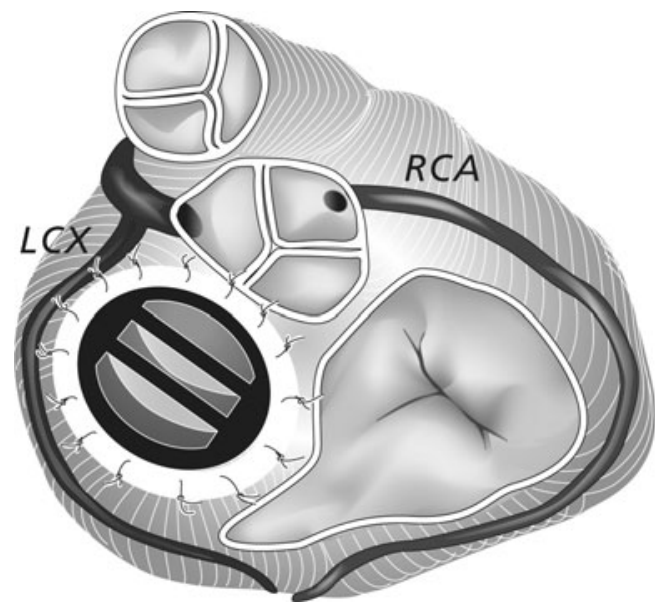

Fig. 6 Cardiac valvular anatomy with a St Jude Prosthetic Heart Valve (PHV) in the mitral position. The illustration demonstrates the close relationship between the LCX and the mitral PHV

In summary, PHV-related artefacts were present in RCA segments (1, 2 and 3), left anterior descending artery (LAD) segment 8 , LCX segments (11 and 13), and MO branches (12 and 14). The close relationship between RCA and aortic PHV, and LCX and mitral PHV is illustrated in Fig. 6. The assessment of the remaining coronary segments (including the left main branch, Fig. 7) was not disturbed by PHVrelated artefacts (Fig. 1, Tables 2 and 3).

\section{Discussion}

The principle findings of our study are: (1) the diagnostic assessment of most coronary artery segments in patients with PHVs was not hampered by PHV-related artefacts, (2) BjörkShiley tilting disc PHVs, Sorin tilting disc PHVs and Duromedics bileaflet PHVs precluded complete diagnostic assessment of coronary artery segments; and (3) ON-X and Medtronic Hall PHVs, biological PHVs and annuloplasty rings never hampered coronary artery assessment by MDCT.

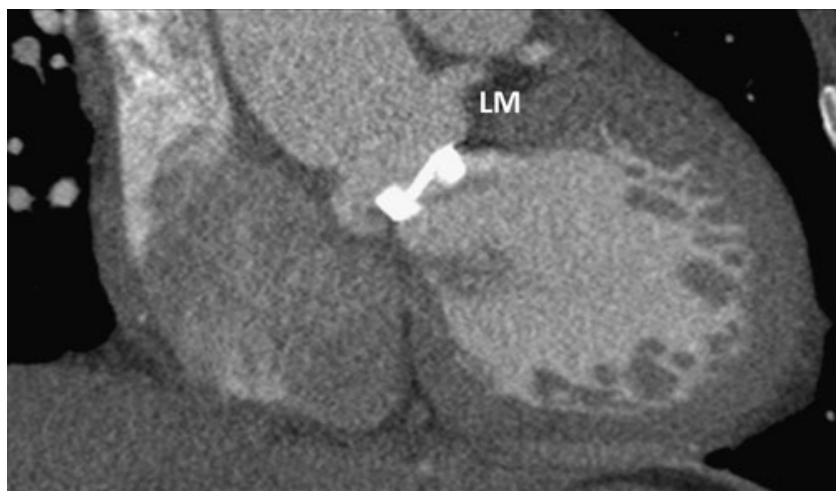

Fig. 7 Carbomedics bileaflet Prosthetic Heart Valve (PHV) in the aortic position, which causes no PHV-related artefacts in the LM branch
The PHV-related artefacts on MDCT images seem to be more dependent on PHV composition than on the PHV design (bileaflet, tilting disc and biological valves) (Table 4) $[8,9]$. As far as the material of the PHV is concerned our study showed notable differences.

Björk-Shiley and Sorin tilting disc PHVs as well as Duromedics bileaflet PHVs demonstrated severe artefacts which precluded diagnostic coronary assessment of RCA, LCX and the distal segment of the LAD, dependent on the PHV position. This finding is supported by previous studies that described that these PHV types as being associated with valve-related artefacts that also prohibit the evaluation of the PHV itself on MDCT $[8,9,11,12]$. The severe artefacts of the Björk-Shiley, Sorin tilting disc and Duromedics bileaflet PHVs were caused by the cobalt-chrome alloy that is present in these valves. Therefore, MDCT is not suitable for the assessment of the coronary arteries in patients who have one of these PHVs implanted. The Saint Jude PHV, consisting of a nickel alloy, produced fewer artefacts but still enough to preclude assessment of a limited number of coronary artery segments in the right coronary artery and circumflex territory (segments 1,13 and 14). The other segments did not suffer from artefacts that precluded assessment. The Saint Jude PHV is one of the most commonly implanted PHV types worldwide and therefore one of the most likely PHVs to be encountered. The possibility of the non-diagnostic image quality of the above-mentioned segments should be kept in mind when performing cardiac $\mathrm{CT}$ in these patients.

In general, Carbomedics, ON-X and Medtronic Hall mechanical PHVs caused no PHV-related artefacts hampering

Table 4 Metallic properties of different prosthetic heart valves (PHV)

\begin{tabular}{lll}
\hline PHV manufacturer & PHV design & Metallic contents \\
\hline Carbomedics & Mechanical bileaflet & Titanium alloy \\
St Jude & Mechanical bileaflet & Nickel alloy \\
ON-X & Mechanical bileaflet & Titanium alloy \\
Sorin bileaflet & Mechanical bileaflet & Titanium alloy \\
Duromedics & Mechanical bileaflet & Cobalt-chrome alloy \\
Medtronic Hall & Mechanical tilting disc & Titanium alloy \\
Sorin monoleaflet & Mechanical tilting disc & Cobalt-chrome alloy \\
Björk-Shiley & Mechanical tilting disc & Cobalt-chrome alloy \\
CE & Annuloplasty ring & Elgiloy \\
CE Perimount & Biological & Elgiloy \\
Sorin Mitroflow & Biological & - \\
Medtronic Mosaic & Biological & Haynes ${ }^{*}$ alloy \\
& & (stent post markers) \\
Medtronic Freestyle & Biological & - \\
St Jude Epic Stented & Biological & Stainless steel wire in \\
& & sewing cuff \\
\hline
\end{tabular}

*Manufacturer data 
coronary artery assessment. These PHVs are mainly composed of titanium alloys that are associated with only limited artefacts on MDCT [8].

The biological PHVs and annuloplasty rings produced fewer PHV artefacts than mechanical PHVs on MDCT. Some biological PHVs have a radiopaque frame that supports the valve leaflets, but this does not generally induce many artefacts $[2,9]$. In our study, no PHV-related artefacts that interfered with diagnostic coronary assessment were found in biological PHVs or annuloplasty rings.

Prosthetic heart valves in the aortic position may show mainly artefacts in the proximal RCA (segments 1 and 2) because of the close relationship between the aortic PHV and the proximal RCA. Interestingly, the left main branch, which also has a close relationship with the aortic PHV, did not show any PHV-related artefacts. The reason for the absence of PHVrelated artefacts in this segment may be the angulation of the PHV with respect to the X-ray beam of the gantry.

In the mitral position, PHV-related artefacts occurred in the LCX and MO branches. The close relationship between these segments and the mitral PHV position is a likely explanation for the presence of the PHV-related artefacts in these specific segments.

\section{Limitations}

Our study contains many different PHV types. A few PHV types are only represented in small numbers. However the most commonly implanted PHVs (Carbomedics, St Jude, Medtronic Hall and the Perimount biological PHVs) are present in considerable numbers. The mean heart rate of $73 \pm 18 \mathrm{bpm}$ is relatively high for CT coronary assessment because 79 of 82 CTAs (96\%) were performed for other clinical indications without a specific heart rate reduction. High heart rates may cause considerable motion artefacts that influence diagnostic CAD assessment. However, the specific interest of this study was to address the question: do PHV-related artefacts disturb coronary assessment? Other reports emphasised the importance of an optimal heart rate and the restricted diagnostic value of MDCT in the detection of CAD in distal coronary segments [13]. To optimize coronary image quality, beta-blockers and nitroglycerin should be routinely administered in patients without contraindications for these drugs. In this study, CT coronary angiography was not compared with conventional coronary angiography. Further prospective studies are required to determine the diagnostic accuracy of CT coronary angiography in patients after PHV implantation. However, as most commonly implanted PHV types generate only limited artefacts, we would expect the diagnostic accuracy to be close to published results in patients who have not yet undergone PHV implantation [13]. Consensus reading was performed. Interobserver variability has to be investigated in further studies to validate the PHV-related artefacts scoring system. Axial CT images only were assessed for the presence of PHV-related artefacts. Multiplanar reconstructions were not separately assessed because PHV-related artefacts were present in both axial and multiplanar reconstructions.

In conclusion, the most commonly implanted PHVs do not cause artefacts that prohibit coronary artery assessment of at least one coronary segment by MDCT. Carbomedics, Medtronic Hall and ON-X mechanical PHVs, bioprosthesis, and annuloplasty rings virtually never hamper coronary artery assessment by MDCT. However, in patients with a Björk-Shiley or Sorin tilting PHV, coronary artery assessment by MDCT is virtually always hampered by PHV induced artefacts.

Acknowledgement We thank K.A. van Rijnbach (Department of Radiology, University Medical Center Utrecht) for her help with the final edition of the Figures and I.G. Janssen (Department of Multimedia Production, University Medical Center Utrecht) for designing Figure 1 and 6. The present study was supported by a grant from the Netherlands Heart Foundation [Grant number 2009B014]

Open Access This article is distributed under the terms of the Creative Commons Attribution Noncommercial License which permits any noncommercial use, distribution, and reproduction in any medium, provided the original author(s) and source are credited.

\section{References}

1. Meijboom WB, Meijs MF, Schuijf JD et al (2008) Diagnostic accuracy of 64-slice computed tomography coronary angiography: a prospective, multicenter, multivendor study. J Am Coll Cardiol 52:2135-2144

2. Chenot F, Montant P, Goffinet C et al (2010) Evaluation of anatomic valve opening and leaflet morphology in aortic valve bioprosthesis by using multidetector CT: comparison with transthoracic echocardiography. Radiology 255:377-385

3. Feuchtner GM, Stolzmann P, Dichtl W et al (2009) Multislice computed tomography in infective endocarditis: comparison with transesophageal echocardiography and intraoperative findings. J Am Coll Cardiol 53:436-444

4. Habets J, Budde RP, Symersky P etal (2011) Diagnostic evaluation of leftsided prosthetic heart valve dysfunction. Nat Rev Cardiol 8:466-478

5. Symersky P, Budde RP, de Mol BA et al (2009) Comparison of multidetector-row computed tomography to echocardiography and fluoroscopy for evaluation of patients with mechanical prosthetic valve obstruction. Am J Cardiol 104:1128-1134

6. Tsai IC, Lin YK, Chang Y et al (2009) Correctness of multidetector-row computed tomography for diagnosing mechanical prosthetic heart valve disorders using operative findings as a gold standard. Eur Radiol 19:857-867

7. Bonow RO, Carabello BA, Chatterjee K et al (2008) 2008 focused update incorporated into the ACC/AHA 2006 guidelines for the management of patients with valvular heart disease: a report of the American College of Cardiology/American Heart Association Task Force on Practice Guidelines (Writing Committee to revise the 1998 guidelines for the management of patients with valvular heart disease). Endorsed by the Society of Cardiovascular Anesthesiologists, Society for Cardiovascular Angiography and Interventions, and Society of Thoracic Surgeons. J Am Coll Cardiol 52:e1-e142 
8. Symersky P, Budde RP, Prokop MA et al (2011) Multidetector-row computed tomography imaging characteristics of mechanical prosthetic valves. J Heart Valve Dis 20:216-222

9. Habets J, Symersky P, van Herwerden LA et al (2011) Prosthetic heart valve assessment with multidetector-row CT: imaging characteristics of 91 valves in 83 patients. Eur Radiol 21:1390-1396

10. Austen WG, Edwards JE, Frye RL et al (1975) A reporting system on patients evaluated for coronary artery disease. Report of the Ad Hoc Committee for Grading of Coronary Artery Disease, Council on Cardiovascular Surgery, American Heart Association. Circulation 51:5-40
11. LaBounty TM, Agarwal PP, Chughtai A et al (2009) Evaluation of mechanical heart valve size and function with ECG-gated 64MDCT. Am J Roentgenol 193:389-396

12. Konen E, Goitein O, Feinberg MS et al (2008) The role of ECG-gated MDCT in the evaluation of aortic and mitral mechanical valves: initial experience. Am J Roentgenol 191:26-31

13. Meijboom WB, Mollet NR, Van Mieghem CA et al (2006) Preoperative computed tomography coronary angiography to detect significant coronary artery disease in patients referred for cardiac valve surgery. J Am Coll Cardiol 48:1658-1665 